\title{
Conceptual design and modeling of a six-dimensional bunch merging scheme for a muon collider
}

\author{
Yu Bao and Gail Hanson \\ University of California, Riverside, California 92521, USA \\ Robert B. Palmer and Diktys Stratakis \\ Brookhaven National Laboratory, Upton, New York 11973-5000, USA
}

(Received 4 September 2015; published 9 March 2016)

\begin{abstract}
A high luminosity muon collider requires single, intense, muon bunches with small emittances: just one of each sign. An efficient front end and a cooling channel have been designed and simulated within the collaboration of the Muon Accelerator Program. The muons are first bunched and phase rotated into 21 bunches, and then cooled in six dimensions. When they are cool enough, they are merged into single bunches: one of each sign. The bunch merging scheme has been outlined with preliminary simulations in previous studies. In this paper we present a comprehensive design with its end-to-end simulation. The 21 bunches are first merged in longitudinal phase space into seven bunches. These are directed into seven "trombone" paths with different lengths, to bring them to the same time, and then merged transversely in a collecting "funnel" into the required single larger bunches. Detailed numerical simulations show that the $6 \mathrm{D}$ emittance of the resulting bunch reaches the parameters needed for high acceptance into the downstream cooling channel.
\end{abstract}

DOI: 10.1103/PhysRevAccelBeams.19.031001

\section{MUON COLLIDER}

The advantages of a muon collider have been reviewed in many publications [1,2]. Muons, because they are 207 times heavier than electrons, synchrotron radiate much less, allowing them, like protons, to be accelerated and collided multiple times in rings. In contrast, high-energy electrons can be accelerated only in linear systems and must be dumped after single collisions. Yet muons, like electrons, are leptons: they undergo simple, single-particle interactions that are much cleaner than those in $\mathrm{p}-\mathrm{p}$ collisions that involve many partons colliding over a wide range of energies.

However, muons, because they are made with very large emittances, must be ionization cooled in all dimensions prior to their acceleration and collision. The required cooling techniques, of which merging can form a part, could also have other applications. They could generate well understood fluxes of both muon and electron neutrinos resulting from muon decays in storage rings with long straight sections [3]. They might also allow fundamental physics studies such as $g-2$ [4] and mu2e [5] experiments with higher statistics.

Figure 1 shows a sketch of a Higgs factory/TeV-scale muon collider designed by the Muon Accelerator Program

Published by the American Physical Society under the terms of the Creative Commons Attribution 3.0 License. Further distribution of this work must maintain attribution to the author(s) and the published article's title, journal citation, and DOI.
(MAP) collaboration. In this configuration muons are first produced indirectly through pion decay by the interaction of a high-power proton beam with a target. Then a set of $\mathrm{rf}$ cavities forms the beam into a string of bunches (21 bunches) and aligns them into nearly equal energies. An ionization cooling channel, which consists of a set of $\mathrm{rf}$ cavities with absorbers, cools the bunches until they can be injected into a bunch-merging system. For muon colliders, because their luminosity is proportional to the square of the number of muons in a bunch, after sufficient initial $6 \mathrm{D}$ cooling, the 21 bunches must be merged into single bunches, one of each sign. After being merged, the single bunches are sent through a second cooling channel where the transverse emittance is reduced as much as possible, and the longitudinal emittance is cooled to a value below that needed for the collider. The bunches with different charges can then be recombined and sent through a final cooling channel [6] using high-field solenoids that cool the transverse emittance to the required values for the collider while allowing the longitudinal emittance to grow. Finally, a series of acceleration stages takes the muon beams to the relevant collider energies.

The emittance evolution in the cooling and merging channels, as required by a muon collider, is shown in Fig. 2. While well-defined concepts for the phase-rotation [7], charge separation [8] and cooling channel [9] subsystems have been previously reported, the conceptual design and simulation of a bunch-merging scheme suitable for a muon collider was far from complete. Therefore, the focus of this paper is to provide a detailed design of the bunch merging 


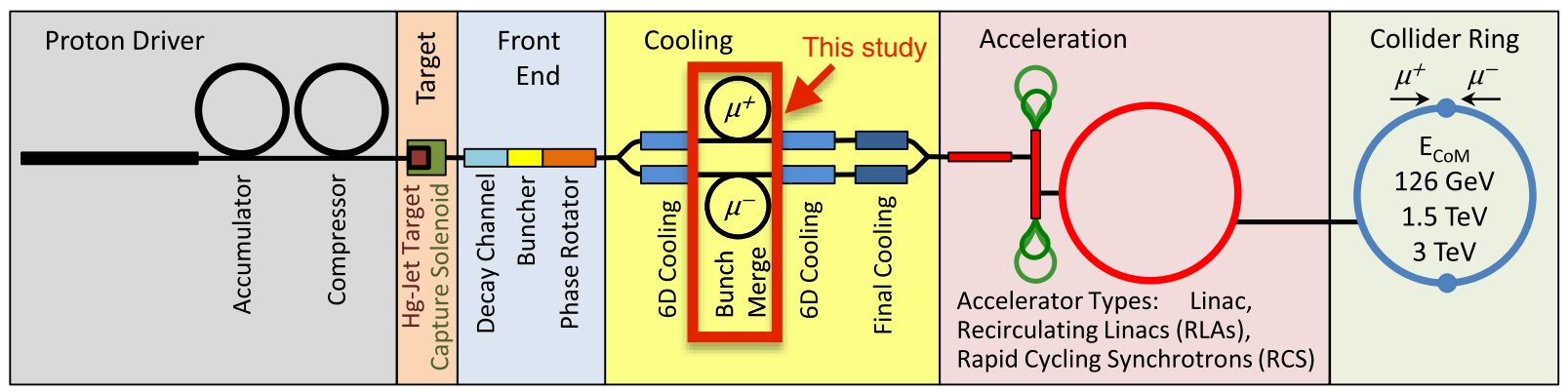

FIG. 1. Functional elements of a muon collider.

system. Before the merge, the 21 bunches are cooled to a transverse emittance $\left(\epsilon_{\perp}\right)$ of $1.3 \mathrm{~mm}$ and a longitudinal emittance $\left(\epsilon_{\|}\right)$of $1.7 \mathrm{~mm}$. After the merge, we require single bunches with transverse emittance of $7 \mathrm{~mm}$ and longitudinal emittance of $10 \mathrm{~mm}$.

In this paper, we investigate the feasibility of merging these 21 bunches into one bunch. Detailed simulations show that the resulting single bunch has a transverse emittance of $6.5 \mathrm{~mm}$ and a longitudinal emittance of $10.3 \mathrm{~mm}$. The total transmission of the scheme is $87 \%$ without decay loss and $78 \%$ with decay. In Sec. II we describe the design and simulation in detail. In Sec. III we present the total performance of the whole bunch merging scenario. In the last section we discuss the challenges and possible improvement of the merging scheme.

\section{MERGE SCHEME}

Figure 3 shows the bunch merge scheme, which includes three major sections:

First, 21 bunches are merged longitudinally into seven bunches, by using radio frequency (rf) cavities with a series of $\mathrm{rf}$ harmonics.

Then the seven bunches are transversely merged into one bunch. A kicker magnet kicks each of the seven bunches into seven different "trombones" [10]. (Only two trombones are

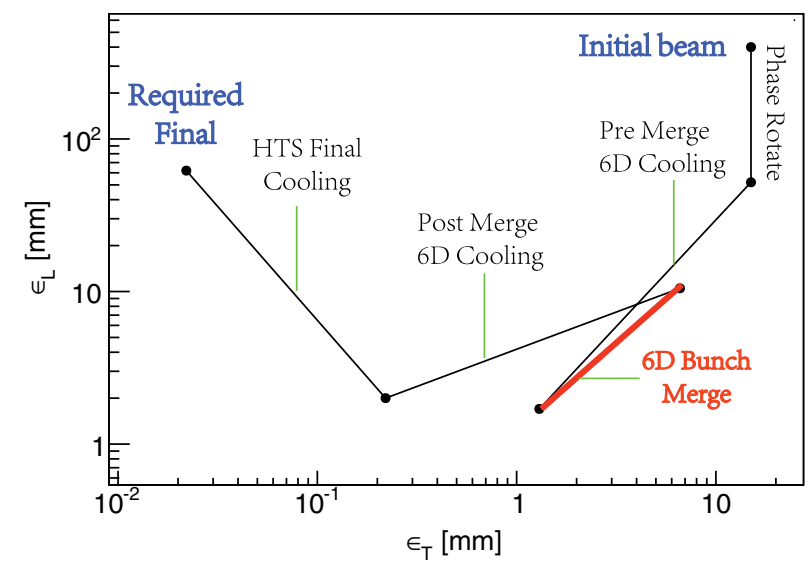

FIG. 2. The longitudinal and transverse emittances during the cooling for a muon collider. shown in Fig. 3.) Each trombone has a different arclength so that seven bunches arrive at the collecting section at the same time. A "funnel" is designed with a pair of dipoles for each bunch to get the seven bunches close to each other to form a single bunch.

In the end a matching section with three solenoids matches the bunch into the postmerge cooling channel.

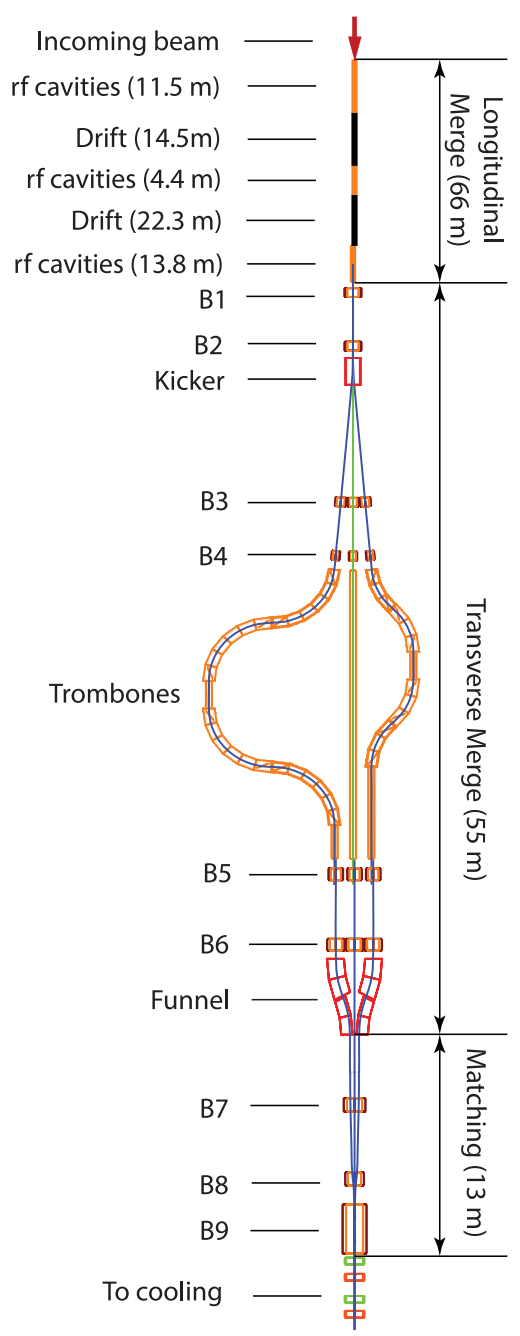

FIG. 3. Merge scheme. 
Such a merge concept was outlined in [11] with preliminary simulations. In this paper we present for the first time an end-to-end simulation with optimized design. Compared to the previous work, a comprehensive study has been done in this paper. First, the previous work used only a 1D simulation without considering chromatic effects. In this paper we present for the first time a 6D simulation. Second, the previous work used an rf frequency of $201 \mathrm{MHz}$, which would not match the requirement of the current baseline design of a muon collider. In this work we use the rf frequencies that fit the current design well. Third, no matching sections were designed in the previous work. We designed the matching sections and developed a numerical method to optimize the matching section. Fourth, in the previous work a linearly increasing kicker field was designed to kick the seven bunches in a plane. We present in this paper a rotating kicking field that reduces the required kicking field and introduces less dispersion from chromatic effects. Fifth, The original trombones were designed with four pieces of 90 degree bent solenoid plus straight sections. The required strong bending field introduced a strong chromatic effect, and the transverse emittance increased quickly in the trombones. In this work we use a large bend radius without straight sections, which greatly limits the emittance growth. We note that all simulations were done with G4BEAMLINE [12], which tracks all relevant physical processes (e.g., energy loss, straggling, multiple scattering) including muon decay.

\section{A. Longitudinal merge}

The longitudinal merge uses a series of rf cavities to phase rotate the bunches: the earlier bunch is decelerated and the later bunch is accelerated so that three bunches as a group form one merged bunch. This is realized with a series of $\mathrm{rf}$ harmonics and drift sections in five stages.

The first stage has ten rf cavities, with five harmonics from 108.33 to $541.67 \mathrm{MHz}$. The cavities give an initial kick to the bunches to rotate them in phase space: the later bunch is accelerated and the earlier bunch is decelerated. Figure 4(a) shows the initial muon energies (relative to the reference muon energy of $130 \mathrm{MeV}$ ) versus the time distributions of six representative bunches that are from the premerge cooling channel [9]. The rf field in the first cavity is shown as a blue curve.

Each cavity in the longitudinal merge is $1.15 \mathrm{~m}$ long and the length of the first stage is $11.5 \mathrm{~m}$. For simplicity the rf field is simulated as the sum of five harmonics in a single cavity. The longitudinal phase space distributions of the bunches after the first stage are shown in Fig. 4(b). A tail in the earlier bunch is developed because of the increase of the bunch length. It eventually causes about $2 \%$ beam loss in the longitudinal merge.

The second stage is a $14.5-\mathrm{m}-$ long drift section. This drift section is used to introduce an energy-time correlation for each bunch. The phase space distributions after the drift are

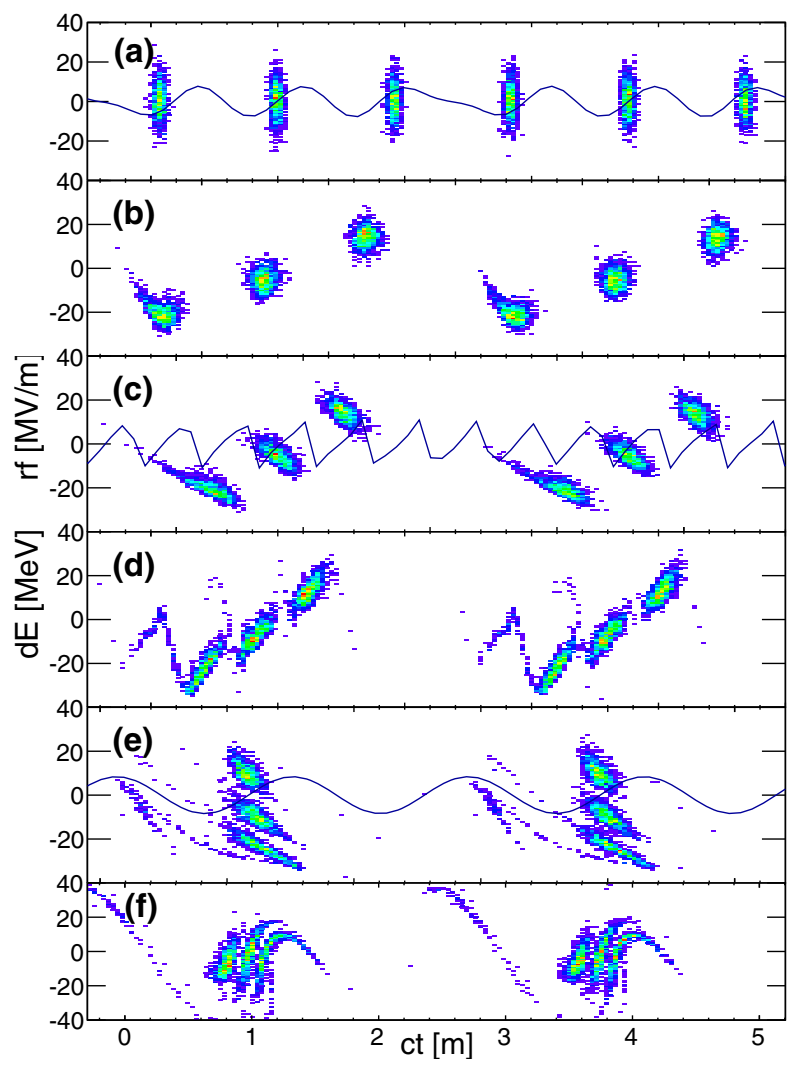

FIG. 4. Phase space distribution at locations along the longitudinal merge: (a)-(e) are at the beginning of each stage and (f) is at the end of the longitudinal merge. The rf fields at the corresponding places are shown by the blue curves. Six representative bunches are shown.

shown in Fig. 4(c). Each bunch is tilted in phase space and has higher-energy muons in the front of the bunch and lower-energy muons at the end.

In order to control the longitudinal phase space of the bunches after the drift section, the third stage uses four $\mathrm{rf}$ cavities to phase rotate the individual bunches. The rf field in this stage is shown as the blue curve in Fig. 4(c). The sawlike field has five harmonics with frequencies from 650 to $1950 \mathrm{MHz}$. It decelerates the muons in the front of the bunch and accelerates the muons at the end. After this stage all the bunches have lower-energy muons in the head and higher-energy muons in the bunch end [Fig. 4(d)].

The fourth stage is a $22.3-\mathrm{m}$-long drift section to get the three bunches closer to the center bunch. During this drift section the higher-energy bunch will catch up with the earlier bunch which has lower energy, and eventually the three bunches will reach the same position/time with different energies. Because the muons in the heads of the bunches have lower energies than muons at the end, the time spread of each bunch is compressed during the drift.

When the three bunches reach the same relative position/ time, as shown in Fig. 4(e), we apply the rf field again to rotate them in phase space. The fifth stage has $12 \mathrm{rf}$ cavities with the same frequency of $216.7 \mathrm{MHz}$ [blue curve in 
TABLE I. Parameters of the bunches after the longitudinal merge.

\begin{tabular}{lclc}
\hline \hline$\sigma_{p}$ & $8.9 \mathrm{MeV} / c$ & $\sigma_{c t}$ & $134 \mathrm{~mm}$ \\
$\sigma_{x}$ & $26 \mathrm{~mm}$ & $\sigma_{x^{\prime}}$ & 0.03 \\
$\epsilon_{L}$ & $9.7 \mathrm{~mm}$ & $\epsilon_{T}$ & $1.4 \mathrm{~mm}$ \\
\hline \hline
\end{tabular}

Fig. 4(e)]. The higher-energy bunch is decelerated and the lower-energy bunch is accelerated until they have approximately the same energy.

At the end of the longitudinal merge, three bunches as a group are merged into one bunch, as shown in Fig. 4(f). The parameters of the resulting bunches are given in Table I. The longitudinal emittance of this bunch is $9.7 \mathrm{~mm}$ with the transverse emittance unchanged. The transmission through the longitudinal merge without considering beam loss from muon decay is $98 \%$. The total length for the longitudinal merge is $66 \mathrm{~m}$ and the decay loss is $4 \%$. In the current paper, the whole longitudinal merge is simulated in a uniform $2 \mathrm{~T}$ solenoidal magnetic field. A more realistic simulation, possibly with quadrupoles, will be done in the future.

\section{B. Kicker and matching section}

After the longitudinal merge, a kicker magnet separates the seven bunches into seven different paths. The ideal design of the kicker would be to kick the seven bunches into seven trombones with different path lengths to bring them back to the same plane at the same time. In order to reduce the complexity of building the geometry in the simulation, in this feasibility study we simplify the scheme to kick six bunches into six trombones and ideally keep the last bunch in the center without being affected. The kicker is a $3-\mathrm{m}$-long magnet with $1 \mathrm{~m}$ diameter. Figure 5 shows the variation of the magnetic field. The magnet has four poles providing magnetic field in both the $x$ and $y$ directions. The transverse field rotates at a frequency of $18 \mathrm{MHz}$, with a field of 220 Gauss to kick the first six bunches by $5^{\circ}$ to six different directions.

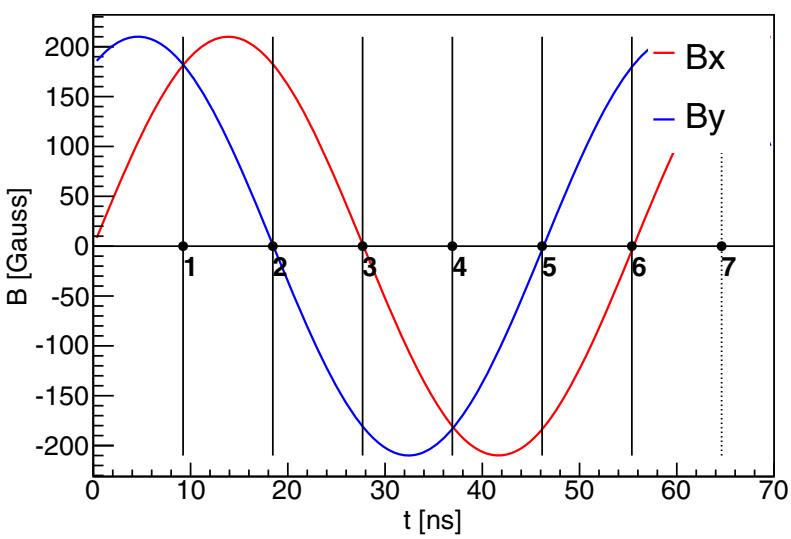

FIG. 5. Rotating magnetic field in the kicker. The transverse magnetic fields $B_{x}$ and $B_{y}$ are shown in red and blue curves respectively.

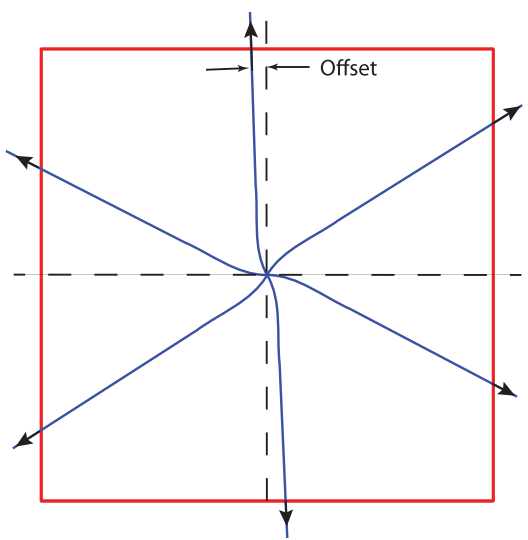

FIG. 6. Cross section of the kicker (red square) with trajectories of the six reference particles of the six bunches (blue lines).

Figure 6 shows a cross section of the kicker with six reference particles representing the trajectories of six bunches. The rotating field kicks the six bunches symmetrically in a circle and introduces an angular and a position offset to the bunches. We rotate the kicker by $1.95^{\circ}$ to compensate for the angular offset, and each bunch is kicked from the centerline by $28 \mathrm{~mm}$ when it reaches the next B3 magnet, and all the following elements in the beam line are aligned to match this offset.

A matching section is designed using four solenoidal magnets (B1, B2, B3 and B4 as shown in Fig. 3) and the kicker is placed between B2 and B3 magnets. The strengths and positions of the four matching solenoids as well as the positions of the entrance of the trombones are optimized by propagating the Courant-Snyder parameters [13]. In a well matched channel, the Courant-Snyder parameters should be maintained at $\alpha(p)=0$ and $\beta(p)=\frac{2 p}{B c}$ for all momenta $p$ of the beam, where $B$ is the magnetic field in T and $c$ is the speed of light in $\mathrm{m} / \mathrm{ns}$. To find such a lattice, we define $\chi^{2}$ :

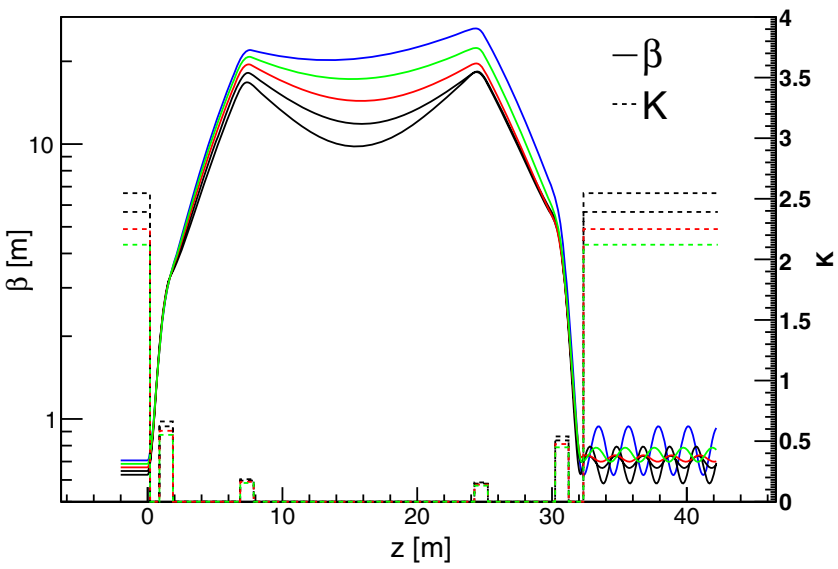

FIG. 7. Optimized effective focusing functions $K(p)$ (dashed lines) and beta functions (solid lines) in the matching channel. Colors represent different muon momenta. 
TABLE II. Parameters for the matching magnets. Positions are the centers of the magnets relative to the end of longitudinal merge.

\begin{tabular}{lccrc}
\hline \hline Magnet & Length & Aperture & Position & \multicolumn{1}{c}{ B } \\
\hline B1 & $1 \mathrm{~m}$ & $1.2 \mathrm{~m}$ & $1.2 \mathrm{~m}$ & $1.2 \mathrm{~T}$ \\
$\mathrm{~B} 2$ & $1 \mathrm{~m}$ & $1.2 \mathrm{~m}$ & $7.2 \mathrm{~m}$ & $0.60 \mathrm{~T}$ \\
$\mathrm{~B} 3$ & $1 \mathrm{~m}$ & $0.8 \mathrm{~m}$ & $24.6 \mathrm{~m}$ & $0.56 \mathrm{~T}$ \\
$\mathrm{~B} 4$ & $1 \mathrm{~m}$ & $0.6 \mathrm{~m}$ & $30.6 \mathrm{~m}$ & $1.0 \mathrm{~T}$ \\
$\mathrm{~B} 5$ & $1 \mathrm{~m}$ & $1.0 \mathrm{~m}$ & $67.5 \mathrm{~m}$ & $1.2 \mathrm{~T}$ \\
B6 & $1 \mathrm{~m}$ & $1.2 \mathrm{~m}$ & $73.5 \mathrm{~m}$ & $0.6 \mathrm{~T}$ \\
B7 & $1 \mathrm{~m}$ & $1.4 \mathrm{~m}$ & $83.6 \mathrm{~m}$ & $0.4 \mathrm{~T}$ \\
B8 & $1 \mathrm{~m}$ & $1.2 \mathrm{~m}$ & $89.1 \mathrm{~m}$ & $0.9 \mathrm{~T}$ \\
B9 & $3.65 \mathrm{~m}$ & $1.5 \mathrm{~m}$ & $92.8 \mathrm{~m}$ & $-2.6 \mathrm{~T}$ \\
\hline \hline
\end{tabular}

$$
\chi^{2}=\sum_{p_{i}}\left[\left[\alpha\left(p_{i}\right)^{2}\right]+\left(\frac{\beta\left(p_{i}\right)-\beta_{0}\left(p_{i}\right)}{\beta_{0}\left(p_{i}\right)}\right)^{2}\right],
$$

where $\beta_{0}$ is the initial Courant-Snyder $\beta$ function before the matching and $\alpha$ and $\beta$ are after matching. Because the mean momentum of the muon bunch is $212 \mathrm{MeV} / c$, and the standard deviation of the momentum distribution is $12 \mathrm{MeV} / c$, we chose $p_{i}=12 i+188 \mathrm{MeV} / c$ where $i=1,2,3,4,5$, and minimize $\chi^{2}$ by varying the strengths and positions of the magnets.

Figure 7 shows the optimized effective focusing function $K$ of the magnets (B1, B2, B3 and B4), where $K=B^{2} c^{2} / 4 p_{i}^{2}$, and the beta functions in the matching channel. Table II gives the parameters of the matching solenoids. The positions are the centers of the magnets relative to the end of the longitudinal merge. The beam is well matched to a $2 \mathrm{~T}$ uniform field. The beta function reaches $0.6 \mathrm{~m}$, which is similar to the incoming beam. The off-momentum muons disperse at the B3 magnet and such dispersion introduces an extra increase of the beam size by about $10 \%$ at the B3 magnet, and it is compressed to $5 \%$ when the bunches are matched to the trombones. The beta function increases to the highest value at the B3 magnet, so a large aperture magnet is needed. The kicker is located at $10 \mathrm{~m}$ from the longitudinal merge, which means the gap between the B2 solenoid and the kicker is $0.8 \mathrm{~m}$. We put the kicker close to B2 and make the distance between B2 and B3 large so that the bunches can be fanned out by the kicker with large enough space to allow seven large aperture solenoids (B3) in parallel. Increasing the beta function at the kicker reduces the angular dispersions of the bunches after being kicked, so that they can transport a long distance to the B3 magnets with an acceptable beam size. Bucking coils are designed for each of these solenoids to avoid the interruption of the fringe fields. Preliminary simulation shows shielding with iron can shield the fringe fields even better.

\section{Trombones}

The seven bunches are separated by $9.23 \mathrm{~ns}$ in time, which means the length difference between the trombones should be
TABLE III. Lengths and bending angles of the trombones.

\begin{tabular}{cccc}
\hline \hline $\mathrm{N}$ & Length/m & $\theta_{N} / \mathrm{rad}$ & $\theta_{N} /$ degree \\
\hline 1 & 34.928 & 1.57 & $90^{\circ}$ \\
2 & 32.464 & 1.46 & $83.7^{\circ}$ \\
3 & 30 & 1.35 & $77.3^{\circ}$ \\
4 & 27.536 & 1.24 & $71.0^{\circ}$ \\
5 & 25.072 & 1.13 & $64.7^{\circ}$ \\
6 & 22.608 & 1.02 & $58.4^{\circ}$ \\
\hline \hline
\end{tabular}

$$
d=\beta c \Delta t=0.267[\mathrm{~m} / \mathrm{ns}] * 9.23[\mathrm{~ns}]=2.464[\mathrm{~m}],
$$

where $\beta c$ is the velocity of the bunch. The longest trombone which guides the first bunch is designed with four $90^{\circ}$ bent solenoids (the first one is $85^{\circ}$ to compensate the kicker angle). By calculating the length difference of this longest trombone and the straight channel, we find the bend radius of this trombone to be $5.559 \mathrm{~m}$ to bring the bunch to the end of the trombone at the same time as the seventh bunch. We use this radius for all the arcs in all trombones, because such a large bend radius requires a small bending field and causes little chromatic effect compared to the small bend radius. The other trombones are designed to have smaller bending angles so that the lengths can fit the separation of the bunches. The $N$ th trombone, where $N$ is the number of the bunch, has a bending angle $\theta_{N}\left(\theta_{1}=\pi / 2\right)$ and radius $R=5.559 \mathrm{~m}$. The length difference of this trombone and the longest trombone should be $(N-1) d$ :

$$
2 \pi R-4 \theta_{N} R=(N-1) d,
$$

where the first term on the left is the length of the longest trombone and the second term is the length of the $N$ th trombone. This equation is used to determine the $\theta_{N}$ for each trombone. Table III gives the lengths and the bending angles of the trombones.

The arcs of the trombones consist of bent solenoids with dipole magnets outside. The bent solenoids have $2 \mathrm{~T}$ field along the centerline, keeping the bunches focused. According to the magnetic rigidity, the dipole field is $B_{d}=3.3356 p[\mathrm{GeV} / c] / e R[\mathrm{~m}]=0.127 \mathrm{~T}$. The distance from the entrance of the trombone to the B4 magnet is $1.6 \mathrm{~m}$, which is also optimized in the matching.

In the middle of the trombones, there are 3-m-long straight sections with $\mathrm{rf}$ cavities to reduce the growth of the longitudinal size of the bunches. The cavities have a frequency of $108 \mathrm{MHz}$ with a gradient of $6 \mathrm{MV} / \mathrm{m}$ and are put inside the $2 \mathrm{~T}$ solenoids. Such a low frequency is used to accept most of the muons longitudinally, and the low gradient is used to avoid breakdown in the magnetic field.

Figure 8 shows the evolution of $\epsilon_{T}$, transmission, and the rms bunch length from the end of the longitudinal merge to the end of the trombone. Here only one channel with the longest trombone is shown, and the others are similar. The magnetic field along the beam line in the beam direction 


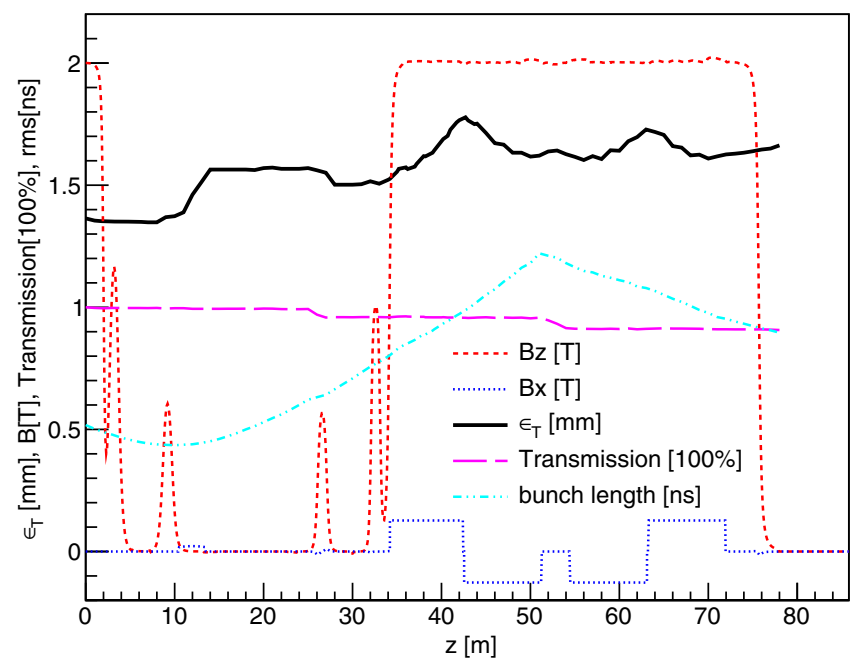

FIG. 8. Evolution of $\epsilon_{T}$, rms bunch length and the transmission from the end of the longitudinal merge to the end of the longest trombone. Both the longitudinal and transverse magnetic fields are shown to indicate the positions of the beam elements.

$\left(B_{z}\right)$ is shown as the red dashed line, and the transverse field is shown as the dotted blue line. The transmission (purple dotted line) without loss from decay is $93 \%$, and the major beam losses are from two places. The first beam loss of about $3 \%$ is caused by the relatively small aperture of magnet B3. The loss of those largely diverged muons also reduces the beam transverse emittance. The second beam loss of $3 \%$ is from the rf cavity in the trombone. The rf field cuts off the muons which have large energy divergence. The beam energy spread is reduced by the cavity, and the longitudinal length also decreases after the rf cavity. The transverse emittance increases at the kicker because of the chromatic effects. For the same reason it increases in the first bent solenoid of the trombone and is corrected by the second inversely bent solenoid. The transverse emittance does not decrease to the same value as before it enters the trombone because the first bent solenoid is $5^{\circ}$ shorter than the second bent section. The transverse emittance $\epsilon_{T}$ also increases and decreases in the third and fourth bend sections but with lower amplitude because the rf cavity in the middle of the trombone reduces the beam energy spread so that the chromatic effects are reduced.

\section{Funnel}

At the end of the trombones the seven bunches are separated by $1.8 \mathrm{~m}$ transversely. A funnel is designed to bring the bunches close and combine them into a single bunch. The bunches first pass through two solenoids (B5 and B6) with bucking coils to reduce the angular dispersion. These two matching solenoids are also optimized by propagating Courant-Snyder parameters to have $\alpha=0$ at the end of the funnel. The parameters of these two solenoid magnets are also included in Table II. The center
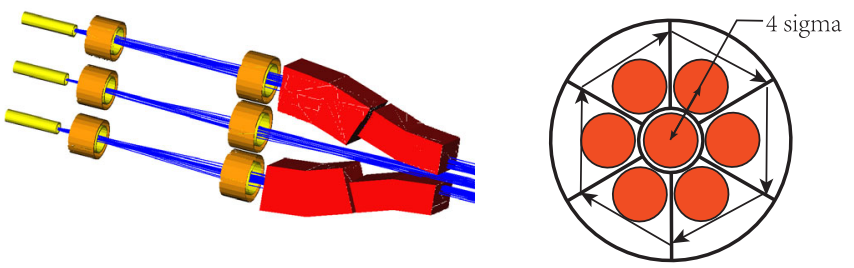

FIG. 9. Left: Schematic of the funnel with three channels shown in detail. Right: Cross section of the seven-channel funnel magnet with dipole fields. Red circles represent the bunches.

bunch still goes straight through the center solenoids. The other bunches pass through the funnel which brings the bunches close to the center bunch. The funnel has six segments. Each segment has a pair of $0.09 \mathrm{~T}$ dipole fields (Fig. 9): the first dipole turns the bunch towards the center and the second dipole turns the bunch back to be parallel to the center bunch. The "septa" of the funnel dipoles are $20 \mathrm{~mm}$ thick and will contain the conductors that generate the uniform bending field. In the current simulation the fringe fields are not included.

In the funnel, we try to get the bunches as close to each other as possible to reach the lowest emittance for the combined bunch. How close they can be brought is limited by beam losses at the septa. The transverse rms radius of each bunch after the funnel is $100 \mathrm{~mm}$. The center bunch radius is $80 \mathrm{~mm}$. In order to get the highest transmission within the acceptable $\epsilon_{T}$, we chose the radius of the center tube to be $200 \mathrm{~mm}$ and the off-center bunches are $400 \mathrm{~mm}$ away from the center.

Figure 10 shows the transverse distribution of the combined bunch after the funnel. The bunch has a large transverse size with $\beta=23 \mathrm{~m}$. The funnel has an efficiency of $94 \%$ and most of the losses are due to the septum magnet. $\epsilon_{T}$ of the combined bunch is $6.6 \mathrm{~mm}$, which is within the acceptance of the later cooling section.

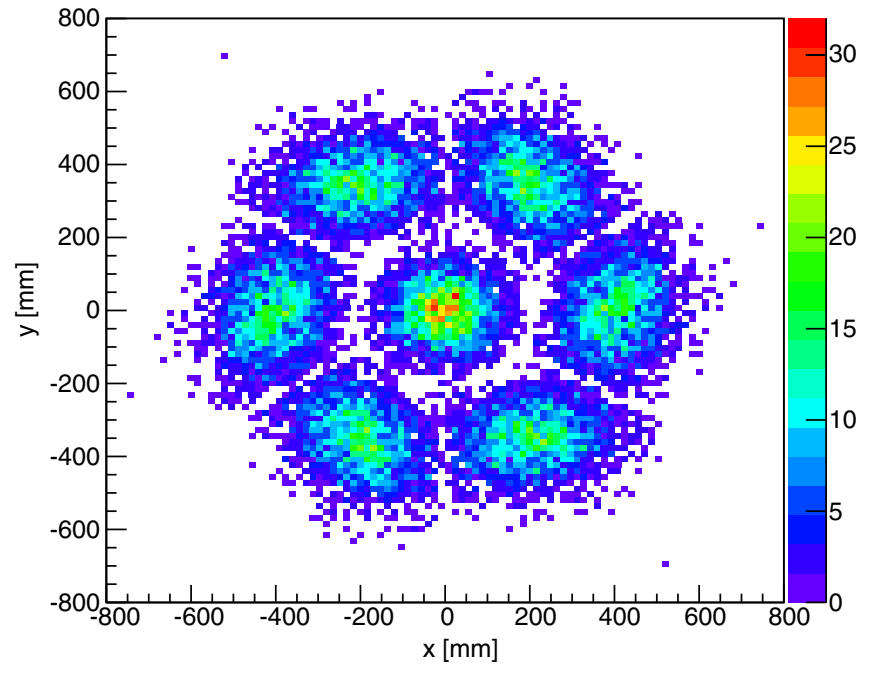

FIG. 10. Beam spatial distribution after the funnel. 


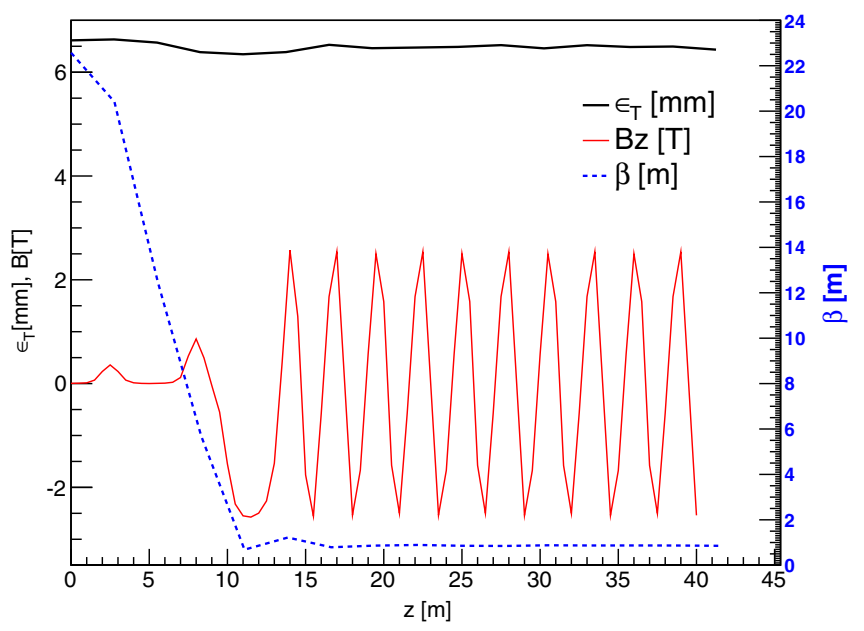

FIG. 11. Transverse emittance along the beam line in the matching section and the first part of the later cooling section.

\section{E. Matching to postmerge cooling section}

After being merged into a single bunch, the bunch has a large transverse size and has to be matched into the later cooling section. The postmerge cooling scheme uses rf cavities with wedge shaped retarding materials in an alternating magnetic field. A matching section is designed with two short solenoidal magnets (B7 and B8) to reduce the transverse size and another $3.65 \mathrm{~m}$ long solenoid (B9) to match the bunch into the alternating field. The strengths and positions of the B7 and B8 solenoids are optimized to reach the minimum $\chi^{2}$ in Eq. (1) at the B9 solenoid. The B9 solenoid has a magnetic field of $2.6 \mathrm{~T}$ in the $-z$ direction and the length of $3.65 \mathrm{~m}$ is optimized to maintain the $\epsilon_{T}$ in the later alternating field.

Figure 11 shows the $\epsilon_{T}$ and $\beta$ function propagating along the matching section. The $\epsilon_{T}$ converges at around $6.5 \mathrm{~mm}$ and the $\beta$ function is reduced to $0.6 \mathrm{~m}$, which is preferable for the cooling channel. In this bunch merging study no cooling process is considered and only the magnetic field in the postcooling channel is used in the simulation for the matching.

\section{TOTAL PERFORMANCE}

Table IV summarizes the bunch parameters before and after the bunch merge. Both $\epsilon_{T}$ and $\epsilon_{L}$ reach the requirements of the later cooling channel. The transmission without decay is $87 \%$ and $78 \%$ with decay. The overall $6 \mathrm{D}$ emittance dilution is about 7 , which can be further reduced, as will be discussed in the next section.

\section{SUMMARY AND OUTLOOK}

Muon beams are commonly produced indirectly through pion decay by interaction of a proton beam with a target. Efficient muon capture requires the muons to be first phase rotated by rf cavities into a train of 21 bunches
TABLE IV. Summary of beam parameters before and after the bunch merge.

\begin{tabular}{lcc}
\hline \hline & Before & After \\
\hline $\mathrm{N}$ bunches & 21 & 1 \\
$x / y[\mathrm{~mm}]$ & 20 & 49 \\
$x^{\prime} / y^{\prime}[\mathrm{rad}]$ & 0.03 & 0.1 \\
$\sigma_{c t}[\mathrm{~mm}]$ & 24.7 & 150 \\
$\sigma_{p}[\mathrm{MeV} / c]$ & 7.51 & 6.5 \\
$\epsilon_{T}[\mathrm{~mm}]$ & 1.3 & 6.5 \\
$\epsilon_{L}[\mathrm{~mm}]$ & 1.7 & 10.3 \\
\hline \hline
\end{tabular}

with much reduced energy spread. Since luminosity is proportional to the square of the number of muons per bunch, it is crucial for a muon collider to use relatively few bunches with many muons per bunch. In this paper we have described a bunch merging scheme that could achieve this goal. According to this scheme, 21 bunches arising from the phase rotator, after some initial cooling, are merged in longitudinal phase space into seven bunches, which then go through seven paths with different lengths and reach the final collecting funnel at the same time to be merged transversely. We show that the final single bunch has a transverse and a longitudinal emittance that matches well into the subsequent $6 \mathrm{D}$ rectilinear cooling scheme with a relatively high transmission. The bunch merging technique described in this paper could have other applications in accelerator physics, such as merging muon bunches for high flux neutrino beams in storage rings and combining multiple short proton bunches on target for pulsed secondary production. There are several possible improvements that can be done in the future to reduce the $6 \mathrm{D}$ emittance dilution.

The longitudinal merge can be further optimized. The frequencies and phases of the rf cavities and the length of the drift sections can be tuned to reach better merge performance.

The kicker introduces a chromatic effect and causes a non-negligible increase of $\epsilon_{T}$. Sextupole magnets can be used to correct this error.

Currently all of the focusing solenoids (B1-B9) are designed with bucking coils to reduce the fringe field transition length. The diameters of the bucking coils are 1.41 times larger than the inner coil aperture. More detailed studies can be done by shielding with iron. Preliminary simulations show that the same thickness of iron shields better than bucking coils, so that using iron can save more space at the B3 magnets and the bending angle at the kicker can be further reduced.

Several technical challenges have to be investigated. The rf harmonics used in the current longitudinal merge simulation have to be replaced by rf cavities. The high frequency kicker field has to be demonstrated. The required space for cooling and the cryogenic system needs to be considered in future work. 


\section{ACKNOWLEDGMENTS}

The authors are grateful to J.S. Berg, H. Witte, K. Yonehara for many useful discussions and suggestions. This work is supported by the U.S. Department of Energy, Contract No. DE-AC02 -07CH11359.

[1] C. M. Ankenbrandt, M. Atac, B. Autin, V. I. Balbekov, V. D. Barger, O. Benary, J. S. Berg, M. S. Berger, E. L. Black, A. Blondel et al. (Muon Collider Collaboration), Status of muon collider research and development and future plans, Phys. Rev. ST Accel. Beams 2, 081001 (1999).

[2] M. M. Alsharo'a, C. M. Ankenbrandt, M. Atac, B. R. Autin, V. I. Balbekov, V. D. Barger, O. Benary, J. R. J. Bennett, M. S. Berger, J. S. Berg et al., Recent progress in neutrino factory and muon collider research within the muon collaboration, Phys. Rev. ST Accel. Beams 6, 081001 (2003).

[3] D. Stratakis and D. V. Neuffer, Compact muon production and collection scheme for highenergy physics experiments, J. Phys. G 41, 125002 (2014).

[4] G. W. Bennett et al. (Muon $g-2$ Collaboration), Final report of the muon e821 anomalous magnetic moment measurement at bnl, Phys. Rev. D 73, 072003 (2006).

[5] D. Brown, The mu2e experiment: Searching for muon to electron conversion, Nucl. Part. Phys. Proc. 260, 151 (2015).

[6] H. Kamal Sayed, R. B. Palmer, and D. Neuffer, High field low energy muon ionization cooling channel, Phys. Rev. ST Accel. Beams 18, 091001 (2015).
[7] C. T. Rogers, D. Stratakis, G. Prior, S. Gilardoni, D. Neuffer, P. Snopok, A. Alekou, and J. Pasternak, Muon front end for the neutrino factory, Phys. Rev. ST Accel. Beams 16, 040104 (2013).

[8] C. Yoshikawa, C. Ankenbrandt, R. P. Johnson, Y. Derbenev, V. Morozov, D. Neuffer, and K. Yonehara, A charge separation study to enable the design of a complete muon cooling channel, in Proceedings of North American Particle Accelerator Conference, Pasadena, CA, 2013 (2013), p. 1343.

[9] D. Stratakis and R. B. Palmer, Rectilinear six-dimensional ionization cooling channel for a muon collider: A theoretical and numerical study, Phys. Rev. ST Accel. Beams 18, 031003 (2015).

[10] G. Flanagan, Using project $\mathrm{x}$ as a proton driver for muon colliders and neutrino factories, in Proceedings of the International Particle Accelerator Conference, Kyoto, Japan (ICR, Kyoto, 2010), p. 4452.

[11] R. Palmer and R. Fernow, Six dimensional bunch merging for muon collider cooling, in Proceedings of the 3rd International Particle Accelerator Conference, New Orleans, LA, 2012 (IEEE, Piscataway, NJ, 2012), pp. 109-111.

[12] T. J. Roberts, K. B. Beard, S. Ahmed, D. Huang, and D. M. Kaplan, G4beamline particle tracking in matterdominated beam lines, in Proceedings of the 24th Particle Accelerator Conference, PAC-2011, New York, 2011 (IEEE, New York, 2011), pp. 3468-3470.

[13] E. D. Courant and H. S. Snyder, Theory of the alternating gradient synchrotron, Ann. Phys. (Paris) 3, 1 (1958). 